\title{
Polyglycolic acid microneedles modified with inkjet-deposited antifungal coatings
}

\author{
Ryan D. Boehm \\ Joint Department of Biomedical Engineering, University of North Carolina and North Carolina State \\ University, Box 7115, Raleigh, North Carolina 27695 \\ Justin Daniels and Shane Stafslien \\ Center for Nanoscale Science and Engineering, North Dakota State University, 1805 Research Park Drive, \\ Fargo, North Dakota 58102 \\ Adnan Nasir \\ Department of Dermatology, University of North Carolina, Chapel Hill, North Carolina 27599 and Wake \\ Research Associates, 3100 Duraleigh Rd. Ste. 304, Raleigh, North Carolina 27612 \\ Joe Lefebvre \\ Hysitron, Inc., 9625 West 76th Street, Eden Prairie, Minnesota 55344
}

Roger J. Narayan ${ }^{\text {a) }}$

Joint Department of Biomedical Engineering, University of North Carolina and North Carolina State

University, Box 7115, Raleigh, North Carolina 27695

(Received 11 January 2015; accepted 9 February 2015; published 2 March 2015)

In this study, the authors examined use of piezoelectric inkjet printing to apply an antifungal agent, voriconazole, to the surfaces of biodegradable polyglycolic acid microneedles. Polyglycolic acid microneedles with sharp tips (average tip radius $=25 \pm 3 \mu \mathrm{m}$ ) were prepared using a combination of injection molding and drawing lithography. The elastic modulus $(9.9 \pm 0.3 \mathrm{GPa})$ and hardness $(588.2 \pm 33.8 \mathrm{MPa})$ values of the polyglycolic acid material were determined using nanoindentation and were found to be suitable for use in transdermal drug delivery devices. Voriconazole was deposited onto the polyglycolic acid microneedles by means of piezoelectric inkjet printing. It should be noted that voriconazole has poor solubility in water; however, it is readily soluble in many organic solvents. Optical imaging, scanning electron microscopy, energy dispersive x-ray spectrometry, and Fourier transform infrared spectroscopy were utilized to examine the microneedle geometries and inkjet-deposited surface coatings. Furthermore, an in vitro agar plating study was performed on the unmodified, vehicle-modified, and voriconazole-modified microneedles. Unlike the unmodified and vehicle-modified microneedles, the voriconazole-modified microneedles showed antifungal activity against Candida albicans. The unmodified, vehicle-modified, and voriconazole-modified microneedles did not show activity against Escherichia coli, Pseudomonas aeruginosa, or Staphylococcus aureus. The results indicate that piezoelectric inkjet printing may be useful for loading transdermal drug delivery devices such as polyglycolic acid microneedles with antifungal pharmacologic agents and other pharmacologic agents with poor solubility in aqueous solutions. (c) 2015 American Vacuum Society. [http://dx.doi.org/10.1116/1.4913378]

\section{INTRODUCTION}

Microneedle arrays have garnered attention over the last two decades as an approach for transdermal delivery of drugs and vaccines. ${ }^{1}$ Microneedles are microscale lancetlike structures that are typically under $1000 \mu \mathrm{m}$ in height. ${ }^{2-5}$ Microneedles are designed to penetrate the stratum corneum layer of skin, which serves as a diffusion barrier for topically applied pharmacologic agents. Permeation of this barrier layer in necessary for delivery of pharmacologic agents to the deeper epidermal layers and dermal layers of the skin.

\footnotetext{
a) Author to whom correspondence should be addressed; electronic mail: roger_narayan@msn.com
}

Hypodermic needles are commonly used for parenteral drug delivery; this approach is often associated with patient noncompliance due to fear associated with needle size and pain; ${ }^{6,7}$ in addition, use of hypodermic needles typically involves administration of the injection by trained personnel. ${ }^{7}$ Although oral drug delivery is often the preferred method for therapeutic agent delivery, first-pass metabolism by the liver and exposure to the gastrointestinal tract can limit the bioavailability of orally delivered drugs. ${ }^{8}$ Microneedles are parenteral devices that may be used for transdermal drug delivery; use of these devices is associated with less discomfort than hypodermic needles. ${ }^{1}$ In addition, microneedles can be fabricated in a patchlike form that can be self-administered by patients. ${ }^{1}$ Microneedles have been investigated for transdermal delivery of many types of small 
molecule drugs (e.g., lidocaine ${ }^{9}$ ), hormones (e.g., insulin ${ }^{10}$ ), and vaccines (e.g., influenza ${ }^{11}$ ).

In this paper, we have investigated fabrication of microneedles out of the polyglycolic acid (PGA) for delivery of the antifungal agent voriconazole. A benefit associated with the use of PGA as a microneedle material is that it is a biodegradable material. In the event of a device failure (e.g., microneedle fracture due to axial or transverse forces during skin insertion), the use of a material that is biocompatible and biodegradable is of interest. PGA has been investigated in the past for the fabrication of microneedles since it is a mechanically robust material. ${ }^{12}$ PGA has been previously utilized as a bioresorbable suture material; ${ }^{13}$ in addition, it has been considered for use in controlled drug release applications ${ }^{14}$ and tissue engineering applications. ${ }^{15}$

Another benefit associated with PGA is that the thermoplastic nature of the material enables it to be processed using the common manufacturing process of injection molding. Lippmann and Pisano studied investment molding of Cyclic Olefin Copolymer (Ticona Topas ${ }^{\circledR}$ ) to create hollow plastic microneedles. ${ }^{16}$ Silicon molds were created with either reactive ion etching or potassium hydroxide etching. Aluminum bond wires were placed within the mold to form the sacrificial investment components. The copolymer material was injected into the molds, encapsulating the investment material. The structure was subsequently exposed to an aluminum etchant to dissolve the investment and leave behind hollow plastic microneedles. Sammoura et al. also utilized the Topas material to create microneedle arrays via injection molding. ${ }^{17}$ Aluminum molds were micromachined into the form of a single microneedle, with an open trench leading to a reservoir cavity at the base of the structure. Another study involves fabrication of microneedles out of poly(lactic acid) (PLA) using an injection molding approach. ${ }^{18}$ Wet-etched silicon molds were created that contained rectangular microneedles with beveled tips. The injection molded PLA microneedles were laser ablated with a UV excimer laser to create either thin trenches along the microneedle exterior surface or to create conduits through the center of the microneedle structure. The trenches and conduits were designed to facilitate blood sampling via capillary action. Park et al. utilized a modified injection molding process to create PLA, PGA, and polylactic- $\mathrm{co}$-glycolic acid microneedles. ${ }^{12}$ Poly(dimethylsiloxane) rubber molds were created from beveled-tip, chisel-tip, and tapered-cone master structures. Pellets of the polymeric material were applied to the mold, introduced to a heated chamber, and exposed to a vacuum, which drew the material melts into the micromold cavities and formed the microneedle arrays.

Another fabrication process, termed drawing lithography, has been utilized to create microneedle structures out of thermoplastic materials; this approach involves controlling the shifts between the glass transition and melting temperatures of the material. ${ }^{19}$ This process utilizes a melt-drawing technique to taper a thermoplastic material into high aspect ratio microstructures. Microneedle arrays have been fabricated out of maltose in this manner for delivery of ascorbic acid and niacinamide. ${ }^{20}$ Another study utilized drawing lithography of maltose on an electrode to create hybrid microneedle-electrodes for cutaneous gene transfer in a murine subcutaneous tumor model. ${ }^{21}$ The drawing lithography process has also been utilized with thermosetting materials (e.g., SU-8 epoxy) to create hollow microneedle structures. 22,23

In the present study, we used a combination of injection molding and drawing lithography to fabricate microneedle arrays out of PGA. Injection molded PGA microstructures were modified into an array of microneedles using an automated drawing lithography procedure. In particular, the drawing lithography approach served to "sharpen" the structures by tapering the features and decreasing the tip radii. The microneedle arrays then underwent a piezoelectric inkjet printing process to apply the antifungal drug voriconazole to the surfaces of the microneedles. This approach to apply coatings to microneedle arrays has been previously demonstrated by our group. ${ }^{24-26}$ Inkjet printing is a noncontact printing technique that may be utilized to precisely apply drug coatings in desired quantities to targeted locations on the surfaces of microneedles and other medical devices. The microneedle arrays were evaluated using optical microscopy, scanning electron microscopy (SEM), energy dispersive $\mathrm{x}$-ray spectrometry (EDS), and Fourier transform infrared spectroscopy. The skin penetration functionality of the microneedles was examined using cadaveric porcine skin and the antimicrobial activity of the microneedles was assessed with an agar plating study.

\section{MATERIALS AND METHODS}

\section{A. Injection molding}

Pellets of Kuredux ${ }^{\circledR}$ PGA (Kureha, Tokyo, Japan) were injection molded into microneedle arrays with steel molds using a Sesame molding machine (Trinks, Inc., De Pere, WI, USA). The melt temperatures for the block and nozzle were set at 227 and $238^{\circ} \mathrm{C}$, respectively. The fixed mold was maintained at a temperature of $41^{\circ} \mathrm{C}$, and the moving mold was maintained at a temperature of $29^{\circ} \mathrm{C}$. The injection speed was $160 \mathrm{~mm} \mathrm{~s}^{-1}$; an injection pressure of $13.79 \mathrm{MPa}$, an initial hold $(0.4 \mathrm{~s})$ pressure at $5.17 \mathrm{MPa}$, a secondary hold $(3 \mathrm{~s})$ at $2.69 \mathrm{MPa}$, and a cooling time of $11 \mathrm{~s}$ were used in this study.

The microneedles were modeled as half-conical structures in a $1 \times 4$ array atop a rectangular base. The center-to-center spacing of the microneedles was set to $1800 \mu \mathrm{m}$. The flat face of the half-cone structure was aligned to be coplanar with one face of the rectangular base [Figs. 1(a) and 1(b)]. The alignment of the microneedles on the base was meant to facilitate the line-of-sight inkjet deposition procedures.

The microneedle arrays produced through the injection molding procedure were imaged with an EZ4D stereomicroscope (Leica Microsystems Inc., Buffalo Grove, IL, USA) to assess microneedle height and tip radii. Measurements of stereomicroscopy data were undertaken using Photoshop CC software (Adobe Systems Inc., San Jose, CA, USA). 

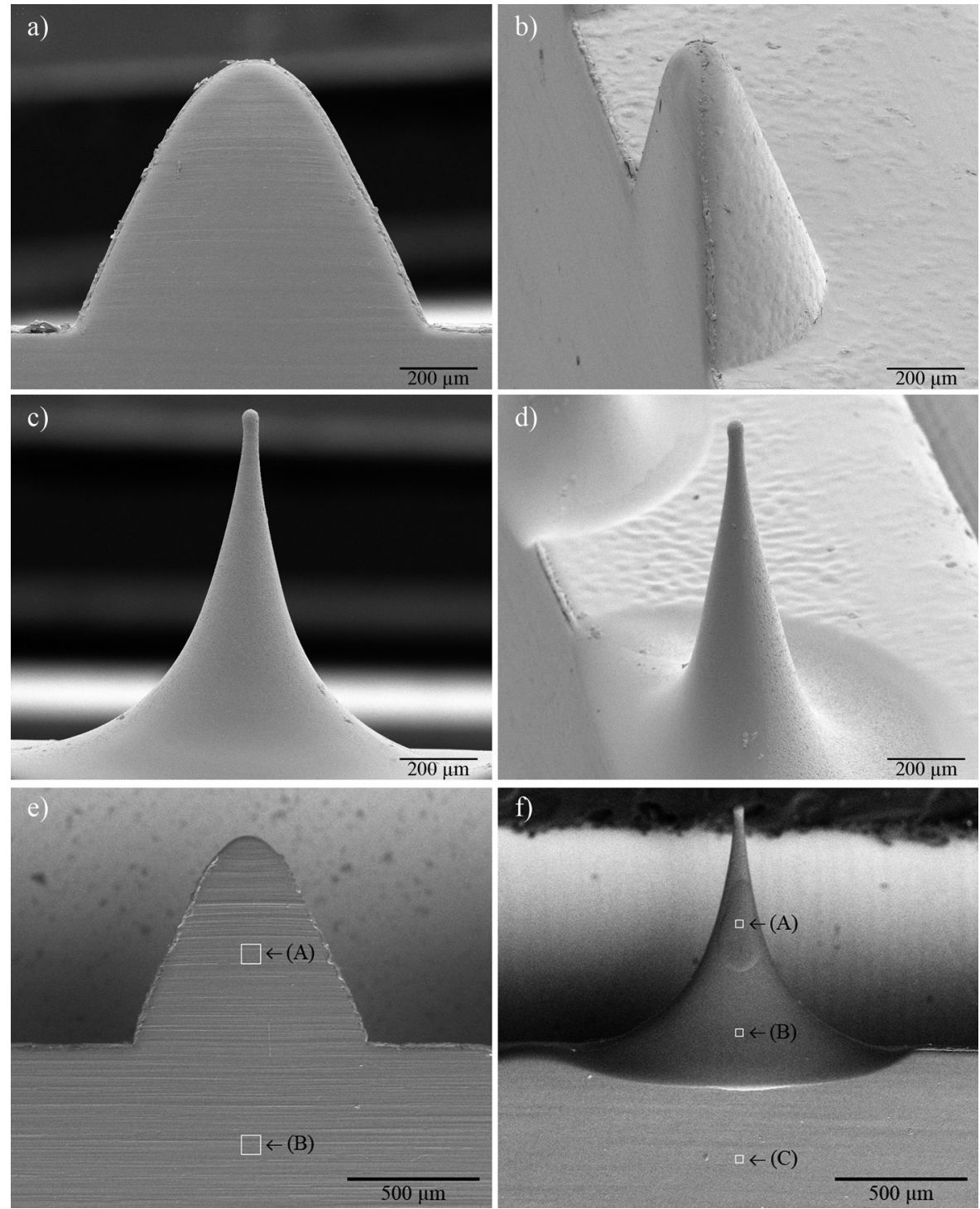

FIG. 1. Scanning electron micrographs of (a) and (b) injection molded PGA microstructures; (c) and (d) drawing lithography-modified PGA microneedles; (e) EDS probe locations (A-microneedle; B-bulk substrate) on an injection molded microstructure; (f) EDS probe locations (A—microneedle inkjet printingmodified zone; $\mathrm{B}$-microneedle noninkjet printing-modified zone; $\mathrm{C}$-bulk substrate).

\section{B. Nanoindentation of injection molded samples}

To assess the hardness and elastic modulus properties of the injection molded PGA materials that had undergone the drawing lithography process, nanoindentation of the samples was conducted with a TriboIndenter TI $950^{\circledR}$ (Hysitron, Inc., Minneapolis, MN, USA). Testing was conducted on the rectangular base of the devices with nine, $5 \mu \mathrm{m}$ long indentations in a $3 \times 3$ grid over a $50 \mu \mathrm{m} \times 50 \mu \mathrm{m}$ square area; a single crystal diamond tip with Berkovich geometry was utilized for indentations. Loads of $8500 \mu \mathrm{N}$ were applied over $5 \mathrm{~s}$, held for $10 \mathrm{~s}$, and released over $1 \mathrm{~s}$, a relatively high load used in this study to minimize the effect of surface roughness on the results. The Oliver-Pharr method was used for determining indentation hardness and elastic modulus of the material by taking into account the indenter tip geometry and calculating the tangential slope of the indentation load-displacement curve ${ }^{27}$ a Poisson's ratio of 0.3 was used for determination of elastic modulus values.

\section{Drawing lithography sharpening on injection molded samples}

The injection molded microstructures were sharpened using a drawing lithography procedure; this process served to reduce the tip radii and increase the aspect ratios of the microstructures. The injection molded microstructures were modified with a hotplate melt-drawing process. The Cimarec ${ }^{\mathrm{TM}}$ hotplate (Barnstead International, Dubuque, IA, USA) was immobilized on an AVS125 servomotor lift platform that was controlled by A3200 software (Aerotech, Inc., Pittsburgh, PA, USA). For each melt-drawing procedure, a microneedle array was attached to a small stainless steel block with double-sided adhesive and suspended in a stationary position above the hotplate; the microneedles were 
pointed toward the heated surface $\left(230^{\circ} \mathrm{C}\right)$ of the hotplate. The hotplate was raised until it made contact with the tips of the microneedle arrays, as confirmed visually with a CM1USB digital microscope (Califone International, Inc., San Fernando, CA, USA). Movement of the microneedle array was paused for $20 \mathrm{~s}$, allowing the microneedle tips to begin melting and the bulk of the material to heat above the glass transition temperature $\left(\mathrm{T}_{\mathrm{g}}\right)$; the $\mathrm{T}_{\mathrm{g}}$ and melting temperature $\left(\mathrm{T}_{\mathrm{m}}\right)$ of Kuredux PGA are 40 and $220^{\circ} \mathrm{C}$, respectively. Following the pause, the hotplate was raised by a value of $550 \mu \mathrm{m}$ at a rate of $0.5 \mathrm{~mm} \mathrm{~min}^{-1}$, which facilitated melting of microneedles within the microneedle array. Movement of the microneedle array was again paused for $3 \mathrm{~s}$ and the hotplate was switched off. The stage was reversed in its z-axis motion; movement of the stage at a rate of $1.0 \mathrm{~mm} \mathrm{~min}^{-1}$ to a distance of $1.25 \mathrm{~mm}$ was undertaken. As the stage motion was reversed, the microneedles were drawn into sharper tapered structures. Cooling and solidification occurred from the base of the structure toward the tip as the PGA material returned below its $T_{m}$. The hotplate was then moved away from the drawing lithography-modified microneedles to enable removal of the microneedle array from the block.

\section{Piezoelectric inkjet printing onto microneedle arrays}

Inkjet printing onto the surfaces of the microneedle arrays was conducted with a Dimatix DMP-2831 material printer (Fujifilm Dimatix, Santa Clara, CA, USA) and a DMCLCP11610 inkjet cartridge (Fujifilm Dimatix, Santa Clara, CA, USA). Three different materials were prepared as the inks that were deposited onto the microneedle surfaces. The first material consisted of a vehicle solution that would later be utilized for the dissolution of voriconazole or methylene blue. The vehicle solution consisted of a copolymer of poly(methyl vinyl ether-co-maleic anhydride) (PMVE/MA), known by the trade name Gantrez AN-119BF (Ashland, Inc., Wilmington, DE, USA). The vehicle solution was dissolved into ACS reagent grade dimethyl sulfoxide (DMSO) (Thermo Fisher Scientific, Waltham, MA, USA) at $5 \%(\mathrm{w} / \mathrm{v})$. The copolymer was added to the DMSO as a thickening agent that served to improve droplet formation during the inkjet printing process and thereby improve the reproducibility of the inkjet printing process. Gantrez AN-119BF was added to DMSO and sonicated for $1 \mathrm{~h}$ to facilitate dissolution. A second solution was created by dissolving methylene blue dye $(5 \mathrm{mM})$ into the vehicle. This solution was utilized to facilitate visualization of penetration by the microneedles arrays into porcine skin. Lastly, a drug solution was created by loading the vehicle solution to $5 \%(\mathrm{w} / \mathrm{w})$ with the antifungal agent voriconazole (Selleck Chemicals, Houston, TX, USA).

A separate DMCLCP-11610 cartridge was utilized for inkjet deposition of each solution onto the microneedle arrays. At least $1.5 \mathrm{ml}$ of solution was loaded into a cartridge using a blunt-tipped syringe. The DMCLCP-11610 cartridges nominally eject $10 \mathrm{pl}$ droplets. In order to calculate the amount of drug being dispensed with each droplet, the drop volume measurement tool within the Dimatix Drop Manager Program was utilized. A preweighed weigh pan was placed under a cartridge loaded with the $5 \%(\mathrm{w} / \mathrm{w})$ voriconazole solution. $5.004 \times 10^{6}$ droplets were ejected at a velocity of $7 \mathrm{~m} \mathrm{~s}^{-1}$ into the pan. Using this approach, the mean weight of each droplet as well as the mass of voriconazole being applied to the microneedles from each droplet was calculated. This calculation was used as a basis for determining the number of droplets to apply to each microneedle in the array. A print pattern was created to apply $0.25 \mu \mathrm{g}$ of voriconazole to each microneedle in the array, totaling $1.0 \mu \mathrm{g}$ of drug for the entire array. It should be noted that $1.0 \mu \mathrm{g}$ is the standard disk diffusion value for assessing quality control of the antifungal characteristics of voriconazole in agar plating assays. $^{28}$

Microneedle arrays were adhered onto glass microscope slides with double-sided adhesive, which facilitated positioning of the conical axes of the microneedles parallel to the printer platen and the printhead. The voriconazole solution was ejected in three layers onto the surface of each microneedle. An optimized waveform was utilized for droplet ejection from two nozzles; a cartridge temperature of $37^{\circ} \mathrm{C}$, an interlayer delay of $120 \mathrm{~s}$, a firing frequency of $2 \mathrm{kHz}$, a voltage tuned to provide $7 \mathrm{~m} \mathrm{~s}^{-1}$ droplet firing velocity, and a droplet-to-droplet spacing value of $5 \mu \mathrm{m}$ were used in this study. Microneedle arrays that were utilized as vehicle controls for antimicrobial testing and microneedle arrays with methylene blue coatings that were utilized for porcine skin penetration analysis were prepared in a similar manner and with the same printing parameters; fresh cartridges loaded with the appropriate ink solutions were used to prepare the vehicle controls and the methylene blue-coated microneedles.

\section{E. Microscopy and energy dispersive x-ray spectrometry}

Microneedle arrays before and after drawing lithography as well as unmodified microneedle arrays and inkjet printingmodified microneedle arrays were examined with SEM and EDS. A S-3200 variable pressure scanning electron microscope with an EDS spectrometer (Hitachi, Tokyo, Japan) was utilized for imaging the microneedles and acquisition of EDS spectra. Samples for imaging were sputter-coated with approximately $14 \mathrm{~nm}$ of $60 \%$ gold- $40 \%$ palladium in a Technics Hummer II system (Anatech, Battle Creek, MI, USA). Imaging was conducted at $5 \mathrm{kV}$ and $90 \times$ magnification. EDS spectra were acquired from uncoated microneedle arrays at several probe locations to examine the inkjet printing-modified zones and the unmodified regions. Samples from injection molding, drawing lithography without inkjet printing modification, vehicle inkjet printing modification, and voriconazole inkjet printing modification were examined with EDS. EDS data were acquired in charge reduction mode with accelerating voltages of 10 and $20 \mathrm{keV}$.

The microneedle arrays were also imaged with a VHX-5000 optical microscope (Keyence, Itaska, IL, USA). 
Optical microscopy was used to examine the inkjet printingmodified regions as well as to measure the heights and tip radii of the lithography-drawn microneedles. The VHX-5000 communication software (Keyence, Itaska, IL, USA) was utilized to obtain measurements from microneedle images that were obtained at $150 \times$ magnification.

\section{F. Fourier transform infrared spectroscopy}

The chemical composition of PGA coated with voriconazole solution by means of inkjet printing was examined using Fourier transform infrared analysis (FTIR). A $1.5 \mathrm{~mm}$ $\times 1.5 \mathrm{~mm}$ square pattern of voriconazole solution was jetted onto a PGA substrate that had undergone the drawing lithography process. The same inkjet printing parameters as described previously were utilized to pattern the voriconazole solution onto the substrate. A FTIR spectrum was acquired from the inkjet printing-modified sample and compared to individual spectra acquired from DMSO, the powder form of Gantrez $^{\circledR}$ AN-119BF, the powder form of voriconazole, and PGA without inkjet printing modification. FTIR data were obtained with a Nexus 470 system, featuring an OMNI sampler (Thermo Fisher, Waltham, MA, USA) and a continuum microscope. Analysis of the spectra was conducted with OMNIC $^{\text {TM }}$ software (Thermo Fisher, Madison, WI, USA).

\section{G. Porcine skin penetration testing}

Penetration of porcine skin by the PGA microneedle arrays was examined with methylene blue-coated microneedle arrays. Full-thickness porcine skin was acquired from a local abattoir and used for testing the same day as received. Prior to testing, the skin samples were allowed to acclimate to room temperature. The microneedles were introduced to the skin by a MiniMed ${ }^{\circledR}$ Quick-serter (Medtronic, Northridge, CA, USA) spring-loaded applicator, which was fitted with a custom adapter to hold a methylene bluemodified microneedle array perpendicular to the skin sample.

Skin samples were trimmed to approximately $5 \mathrm{~cm} \times 5 \mathrm{~cm}$ pieces $(\sim 1 \mathrm{~cm}$ thick $)$, placed in a plastic petri dish, and gently blotted dry with a Kimwipe ${ }^{\mathrm{TM}}$ (KimberlyClark Corporation, Irving, TX, USA). A microneedle array was loaded into the applicator adapter, the spring-loaded plunger was locked into place, and the applicator was placed on the skin surface. Upon release of the springloaded plunger, the microneedles were introduced to the porcine skin. Light finger-pressure was applied to the plunger for $15 \mathrm{~s}$. The array was left in place in the tissue for 15 min to facilitate dissolution of the methylene blue coating. Following removal of the array, the insertion sites were swabbed once with $0.85 \%$ normal saline on a cotton-tipped applicator. The location was then swabbed once with ethanol and once with a dry cotton-tipped applicator. The penetration sites were examined under magnification and imaged with the VHX-5000 optical microscope at $200 \times$ magnification.

\section{H. Antimicrobial testing}

Antimicrobial testing was performed as described previously. ${ }^{5}$ Luria-Bertani broth, tryptic soy broth, Mueller Hinton agar, yeast nitrogen base, Sabouraud dextrose agar, dextrose, agar, and phosphate-buffered saline $(\times 10)$ were obtained from a commercial vendor (VWR International, West Chester, PA, USA). Phosphate buffered saline (PBS) (x1) was prepared by dilution in deionized water. The microorganisms utilized in the study were acquired from a commercial source (American Type Culture Collection, Manassas, VA, USA). The microorganisms included Candida albicans ATCC 90028, Escherichia coli ATCC 12435, Pseudomonas aeruginosa ATCC 15442, and Staphylococcus aureus ATCC 6538. Cultures were prepared overnight with $E$. coli in Luria-Bertani broth, $P$. aeruginosa in tryptic soy broth, $S$. aureus in tryptic soy broth, and $C$. albicans in yeast nitrogen base and $100 \mathrm{mM}$ dextrose. The cultures were pelleted by centrifugation $(4500 \mathrm{rpm})$ for $10 \mathrm{~min}$ and resuspended in PBS $(\times 1)$ to a cell density of $\sim 10^{8}$ cells $\mathrm{ml}^{-1}$. Sterile swabs were used to inoculate agar plates with lawns of the microorganisms. C. albicans lawns were inoculated on Sabouraud dextrose agar plates. The E. coli, $P$. aeruginosa, and $S$. aureus lawns were inoculated on Mueller Hinton agar plates. Visualization was aided by incorporating a redox indicator dye, triphenyltetrazolium chloride, into the agar plates; this dye forms a red color upon microbial growth.

Three sets of microneedle arrays were prepared for antimicrobial testing. Lithography-drawn samples that were either (1) voriconazole-modified, (2) vehicle-modified, or (3) without inkjet printing modification were examined for antimicrobial activity using the modified-disk diffusion, agar plating assay. The microneedle arrays were applied to the inoculated agar plates and incubated for $24 \mathrm{~h}$ at $37^{\circ} \mathrm{C}$. Inhibition of growth on the plates was evaluated from digital images that were obtained following $24 \mathrm{~h}$ of incubation.

\section{RESULTS AND DISCUSSION}

\section{A. Mechanical properties}

Nanoindentation provided hardness and elastic modulus values for the PGA material. It indicated that the PGA material had an indentation hardness value of $588.2 \pm 33.8 \mathrm{MPa}$ (mean \pm standard deviation). The reduced elastic modulus obtained from indentation was $10.9 \pm 0.3 \mathrm{GPa}$ (mean \pm standard deviation). Taking into account the elastic modulus and Poisson's ratio of the diamond indenter tip (1140 GPa and 0.07 , respectively) and assuming a Poisson's ratio of 0.3 for the PGA material, the elastic modulus of the PGA material was calculated as $9.9 \pm 0.3 \mathrm{GPa}$ (mean \pm standard deviation). Park et al. considered the mechanical properties of microneedles and noted that microneedle materials with Young's modulus values higher than $\sim 1 \mathrm{GPa}$ were associated with fracture forces that exceeded skin insertion forces. ${ }^{12}$ Microneedle failure was also shown to be dependent on height and base diameter; tall microneedles $(\geq 1 \mathrm{~mm})$ 
and microneedles with small base diameters $(100 \mu \mathrm{m})$ were noted to be subject to lower fracture force values.

\section{B. Drawing lithography sharpening of injection molded materials}

The structures obtained from the injection molding procedure exhibited rounded tips and intra-array inconsistencies with respect to microneedle height and microneedle tip. The microneedle arrays were initially examined via optical stereomicroscopy. The injection molded structures had heights of $816 \pm 40 \mu \mathrm{m}$ and tip radii of $113 \pm 30 \mu \mathrm{m}$ (mean \pm standard deviation; $n=44)$. There was variation in height and tip radius among the microneedles within the array; a mean intraarray height difference of $80 \mu \mathrm{m}$ between the tallest and shortest microneedle was observed. Adjustments to the processing parameters may be able to more finely regulate the geometries of the structures created through the injection molding process.

The process of heating and melt-drawing the injection molded structures into sharper structures proved to be a facile approach to creating narrower microneedles with sharper tip radii. As mentioned earlier, the injection molded structures had heights of approximately $800 \mu \mathrm{m}$, significant intraarray variability in microneedle height, and average tip radii exceeding $100 \mu \mathrm{m}$. Figures 1(c) and 1(d) provide a scanning electron micrograph that shows the effect of the drawing lithography process. The drawing lithography process produced microneedles with similar intra-array height values, narrower and tapered forms, as well as reduced tip radii.

The microneedle arrays following the drawing lithography process were optically evaluated to determine microneedle heights and microneedle tip radii. The VHX-5000 communication software (Keyence, Itaska, IL, USA) was utilized for optical measurements of the drawn arrays. The microneedles that underwent drawing lithography exhibited were slightly smaller than the injection molded structures that served as the starting materials; the microneedles that underwent drawing lithography exhibited heights of $754 \pm 68 \mu \mathrm{m}$ (mean \pm standard deviation; $\mathrm{n}=36$ ). The microneedles that underwent drawing lithography exhibited smaller tip radii than the injection molded structures that served as the starting materials; tip radii of the microneedles that underwent drawing lithography were $25 \pm 3 \mu \mathrm{m}$ (mean \pm standard deviation; $\mathrm{n}=36$ ). Intra-array variations in microneedle height were noted in microneedle arrays that were prepared using the drawing lithography process. For example, an intra-array positional dependence was noted in the microneedle height and the microneedle tip radius. This observation may be attributed to height differences in the features of the injection molded structure, which contributed to differences in the amount of material being drawn during the lithography process. In addition, there may have been nonuniformity in heating with respect to the peripheral positions versus the central positions in the microneedle array; the central microneedles were typically taller than the microneedles on the periphery. Furthermore, an absence of true planarity of the hotplate surface could introduce some nonuniformity in the drawing procedure.

\section{Piezoelectric inkjet printing modification}

Piezoelectric inkjet printing was used to apply the voriconazole coating onto microneedles in the microneedle arrays. The mean mass of each droplet was determined from the mass of $5.004 \times 10^{6}$ droplets that were inkjet printed into a weigh pan, which was immediately weighed. The average weight of each droplet (10.0 ng/drop) and the mass to each droplet contributed by the voriconazole $(0.5 \mathrm{ng} / \mathrm{drop})$ were calculated from this value. An inkjet printing-deposited pattern was implemented to apply 496 droplets per microneedle; the pattern deposited $1.0 \mu \mathrm{g}$ of voriconazole evenly among the microneedles in the array.

The microneedles were examined with optical microscopy, SEM, as well as EDS to assess the alterations to the microneedles associated with the inkjet printing process. As targeted by the inkjet printing process, the inkjet printing-modified regions were localized to the "upper" half of the microneedles (nearest to the microneedle tips). Figure 2 shows an unmodified and a voriconazole-modified microneedle array. Differences between the inkjet printing-modified microneedles and the unmodified

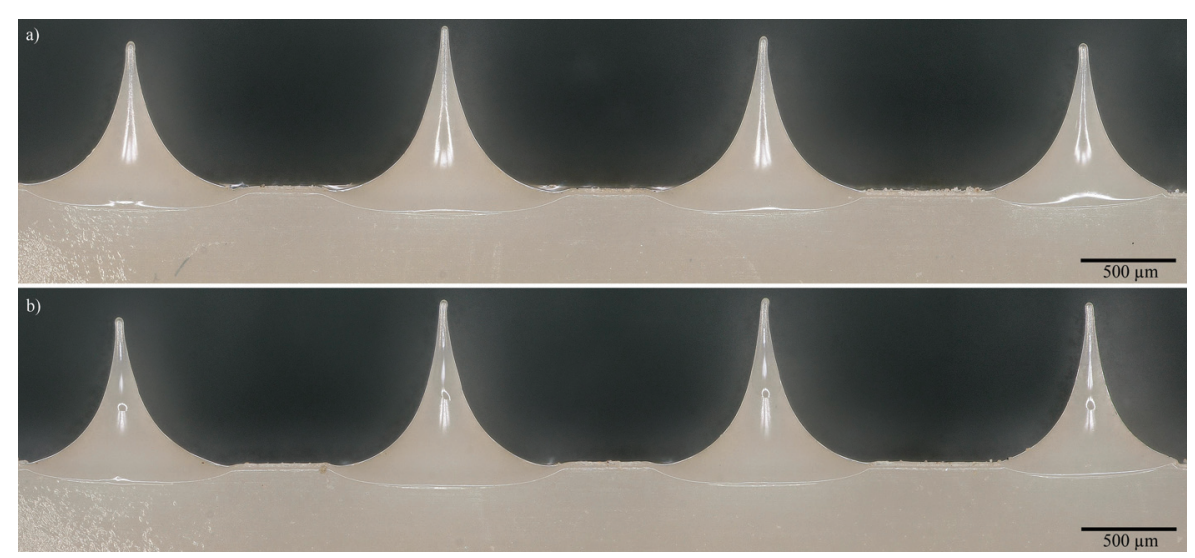

FIG. 2. Optical micrographs of (a) a drawing lithography-modified PGA microneedle array without inkjet modification (b) a drawing lithography-modified PGA microneedle array after inkjet printing of voriconazole. The inkjet modification is discernible in the upper half of the drawing lithography-modified microneedles. 
microneedles were associated with the inkjet printing process. The images suggest that the inkjet printing procedure was able to apply material directly onto the microneedles. In addition, optical observations of the underlying glass substrates to which the microneedles were adhered during the inkjet printing procedure indicated that inkjet printing was localized to the microneedle surfaces; evidence of stray satellite droplets was minimal.

EDS was utilized to examine the inkjet printing-modified zones of the microneedles, the unmodified zones of the microneedles, as well as the PGA bulk material. The injection molded structures were probed on the microneedle surface and the bulk material [Fig. 1(e)], exhibiting carbon and oxygen in both probe locations of the arrays. Similarly, the drawing lithography prepared samples without print modification were probed and exhibited signals for carbon and oxygen. Detection limit quantities of silicon were also noted in the drawn regions but not the bulk material. The presence of this trace amount of silicon was attributed to interactions between the polymer microneedles and the ceramic surface of the hotplate during the drawing lithography procedure. Figure 1(f) shows a diagram of regions that were investigated with the EDS probe on the vehicle- and voriconazolemodified microneedle arrays. On the vehicle-modified microneedles, the probed regions of the bulk material and lower sections of the microneedles indicated the presence of carbon and oxygen. In the microneedle regions that underwent the drawing lithography process, a trace amount of silicon was observed. Spectra of the inkjet printing-modified regions exhibited an additional signal from sulfur. This signal, which was associated with the presence of DMSO in the vehicle solution, was localized to the inkjet printing-modified region of the microneedles. The EDS data for the voriconazolemodified microneedle arrays indicated the presence of carbon and oxygen in the bulk material. The spectra showed carbon, oxygen, and trace amounts of silicon in the drawn sections of the microneedles. In the inkjet printing-modified region, sulfur (from DMSO) as well as fluorine were observed. The fluorine signal in the inkjet printing-modified region was associated with the presence of voriconazole. The inkjet printing-modified region of the voriconazole-modified microneedle arrays were the only probed locations with a fluorine signal, suggesting precise deposition of the voriconazole solution on the microneedle surface. It should also be noted that an observable aluminum signal was noted in the EDS spectra of all of the probed samples. An increased signal was noted at higher accelerating voltages used to acquire the spectra; the signal was more intense when probing the microneedle tips compared to the bulk material. Consequently, the aluminum signal was attributed to backscattering of electrons that had interacted with the aluminum SEM stub and not from the samples themselves.

FTIR spectra obtained from the inkjet printing-modified samples (Fig. 3) contain features that are consistent with the components of the voriconazole-containing inkjet solution and the PGA substrate. A number of features associated with Gantrez AN-119BF can be identified within the spectrum. A broad rounded shoulder from $\sim 3450$ to $3000 \mathrm{~cm}^{-1}$ is associated with an intense hydroxyl signal. ${ }^{5,24,29}$ Carbon-hydrogen stretching peaks are seen at 2937.8 and $2844.7 \mathrm{~cm}^{-1}$. A peak at $2564.8 \mathrm{~cm}^{-1}$ is associated with ether bond cleavage and -OH contributions from carboxylic acid formation following dissolution of the copolymer material..$^{5,24,30}$ The doublet at 1852.5 and $1777.9 \mathrm{~cm}^{-1}$ is characteristic of $\mathrm{C}=\mathrm{O}$ stretching from the carbonyl of the acid anhydride. ${ }^{30-32}$ The characteristic carboxylic $\mathrm{C}=\mathrm{O}$ band of PGA is largely masked by the strong hydroxyl signal in the $\sim 3000-2960 \mathrm{~cm}^{-1}$ region. ${ }^{33-36}$ Characteristic ester $\mathrm{C}=\mathrm{O}$ stretching is observed at the $1716.1 \mathrm{~cm}^{-1}$ peak; however, it is slightly shifted to a lower wavenumber compared to the value reported elsewhere $\left(1744 \mathrm{~cm}^{-1}\right) .^{35,36}$ This shift may be attributable to contributions from the PMVE/MA material. ${ }^{5,24}$ There is also a characteristic $\mathrm{C}-\mathrm{O}$ band at $1100.2 \mathrm{~cm}^{-1} \cdot 32$ Voriconazole was associated with $\mathrm{C}-\mathrm{F}$ stretching contributions in the $1594.8-1457.9 \mathrm{~cm}^{-1}$ range and $\mathrm{C}-\mathrm{N}$ stretching in the $1501.0-1457.9 \mathrm{~cm}^{-1}$ region. ${ }^{37,38}$ Spectral contributions from DMSO were also noted. In particular, symmetric $\left(-\mathrm{CH}_{3}\right)$ bending associated with DMSO was observed at 1435.7 and

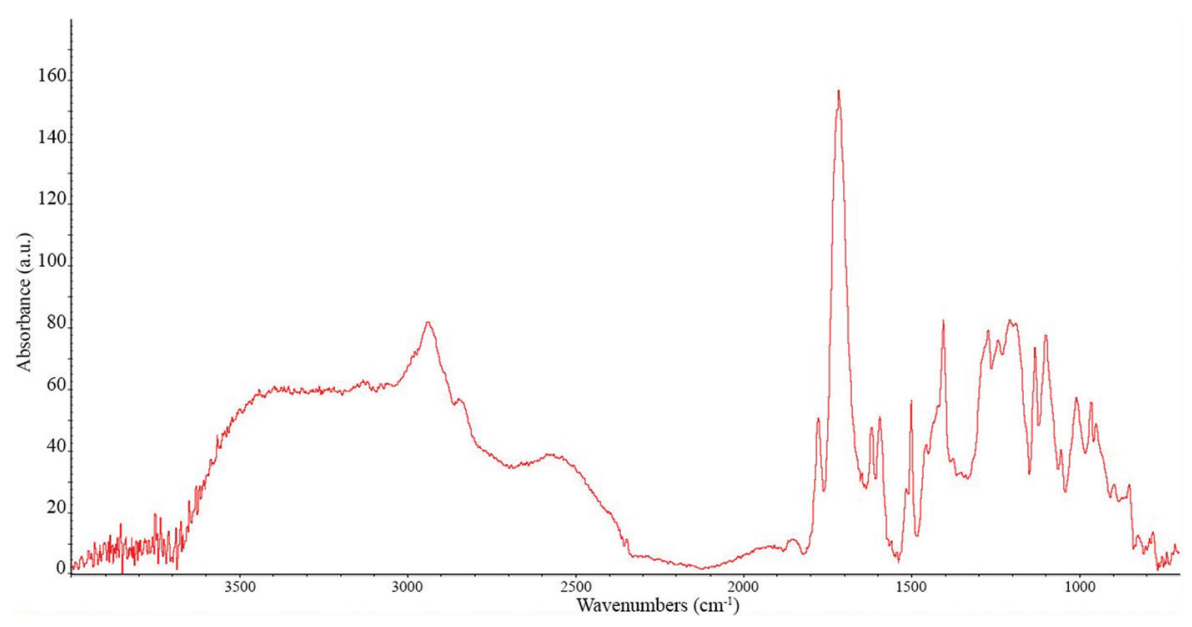

FIG. 3. FTIR spectrum of PGA modified with voriconazole using inkjet printing. 
$1405.7 \mathrm{~cm}^{-1} .39$ A band associated with $\mathrm{S}=\mathrm{O}$ stretching was observed at $1055.3 \mathrm{~cm}^{-1} .{ }^{39}$ Furthermore, the characteristics observed in the composite FTIR spectrum were in agreement with the spectra of the individual components (data not shown), indicating that the inkjet printing process did not appreciably alter the drug solution.

Inkjet printing has been shown in the past to be an effective means of depositing pharmacologic agents. Scoutaris et al. examined the inkjet deposition of felodipine and polyvinyl pyrrolidone (PVP) mixtures onto glass substrates. ${ }^{40}$ Homogeneity was observed in the microspots of the patterned PVP-drug mixtures. Drug release was modified by varying the drug loading percentage within the inks, suggesting the potential to create a controlled release platform with varying concentrations of drug within a printed pattern. In another study, acetaminophen, theophylline, and caffeine were deposited onto pigment-coated paper, uncoated paper, and polyethylene terephthalate film using a Dimatix DMP-2800 piezoelectric inkjet printer. ${ }^{41}$ The study investigated the crystallization of the model drugs as well as penetration into porous substrates as a potential means for creating complex drug delivery systems. The authors noted the potential of this process to facilitate accurate dosing of low-dosage medicine, fabrication of on-demand individualized medicine delivery systems, and a mechanism for delivery of poorly soluble drugs. These concepts are also applicable to microneedle arrays. In this study, microgram quantities of voriconazole were applied to the microneedle surfaces; the dosage of the pharmacologic agent can be adjusted on-demand by altering the printing pattern and deposition parameters. Furthermore, voriconazole is a drug that is poorly soluble in water, necessitating dissolution by DMSO in this printing process.

Inkjet printing has been previously utilized by our group as an approach to apply surface coatings to microneedle arrays. $^{24-26}$ Antifungal pharmacologic agents, including amphotericin B (Ref. 25) and miconazole, ${ }^{26}$ have previously been applied to polymeric microneedles using piezoelectric inkjet printing. This approach provides reproducibility and precision in the quantity and location of the pharmacologic agent that is applied to the microneedle. The inkjet printing process can be readily modified for use with other poorly water-soluble drugs and/or for modification of microneedle arrays with other geometries. Furthermore, the targetspecific nature of the printing process could facilitate preparation of multiplexed microneedle arrays, in which a variety of drugs are deposited onto a single microneedle for use in combinatorial drug therapy. This concept supports the premise of creating sophisticated drug delivery devices that can be personalized for individual patient needs.

\section{Porcine skin penetration}

Microneedle penetration into porcine skin was used assessed using by application of methylene blue-coated microneedles using a spring-loaded applicator. Methylene blue has been utilized in a number of other studies for confirming microneedle penetration into skin. ${ }^{42-45}$ In our study, dissolution of the methylene blue coating was noted on the microneedle surface following application to the skin. Figure 4 shows before [Fig. 4(b)] and after [Fig. 4(c)] images of a methylene blue-coated microneedle. Following

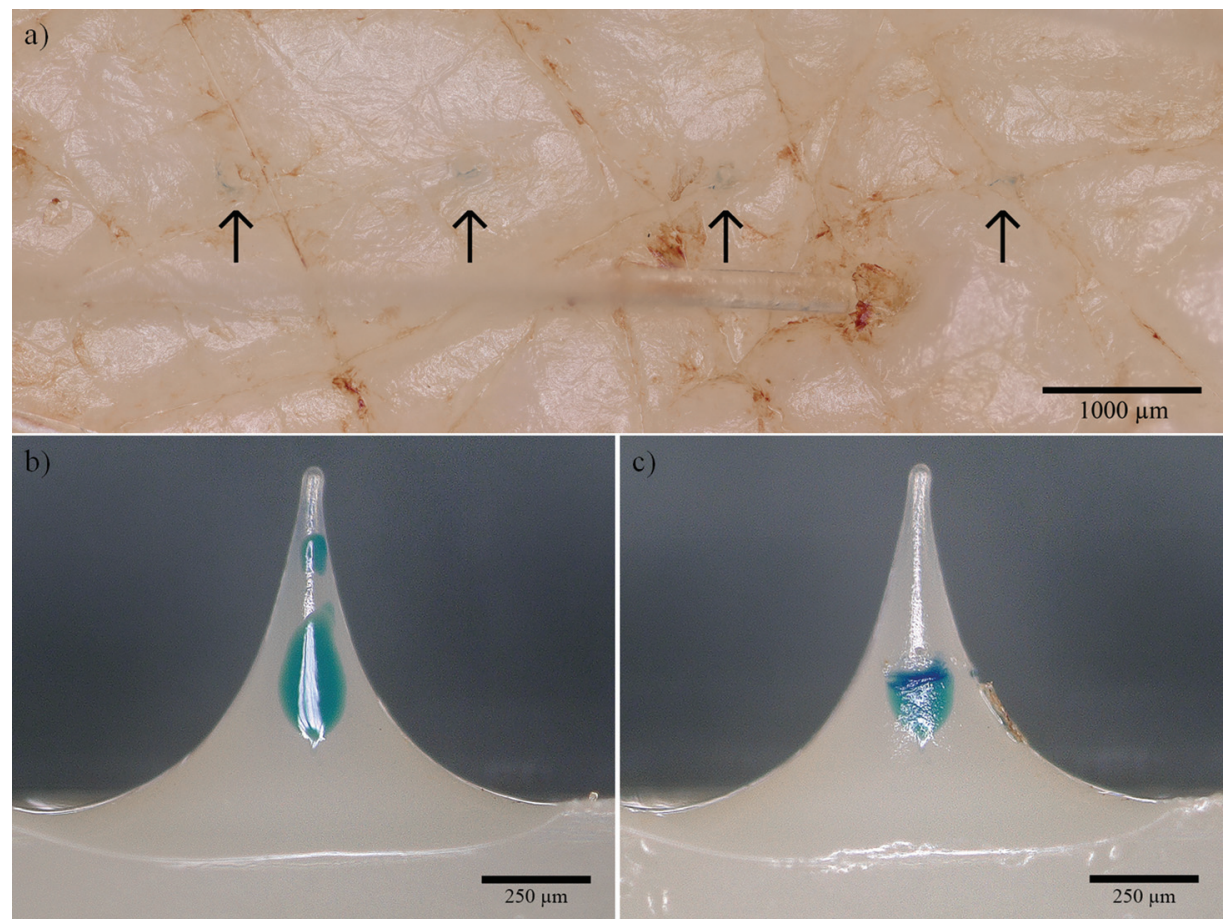

FIG. 4. Optical micrograph of (a) porcine skin following application of a methylene blue-coated microneedle array (arrows indicate puncture locations); (b) methylene blue-coated microneedle prior to skin testing; (c) methylene blue-coated microneedle following skin testing, with dissolution of the blue coating at the upper portion of the microneedle. 
the 15 min application time, the blue-colored coating was not apparent on the upper portion of the microneedle. The penetration test suggests that the upper portion of the microneedle penetrated into the skin, enabling dissolution of the surface coating. Furthermore, the injection sites in the porcine skin exhibited a blue color due to the methylene blue dye; this skin discoloration remained following swabbing of the insertion site with saline and ethanol. Figure 4(a) displays an example insertion site for an array of microneedles. It is apparent that there are four puncture sites within the tissue, which are located in congruence with the tip-to-tip microneedle spacing of the microneedle array. Furthermore, there is a visual confirmation of the localization of the methylene blue dye within the insertion sites and beneath the skin surface. These results are indicative of penetration of the microneedle array through the stratum corneum layer, facilitating release of the dye from the microneedle surface coating into deeper layers of the skin.

\section{E. Antimicrobial testing}

The results of the microneedle interactions with the microbial cultures in the agar plating study are shown in Figs. 5(a)-5(d), with (a) C. albicans, (b) E. coli, (c) P. aeruginosa, and (d) S. aureus. A qualitative assessment can be made regarding the antimicrobial effects of the unmodified, vehicle-, and voriconazole-modified microneedles on the microbial cultures. For each of the tested bacteria, there was no observable inhibition of growth following $24 \mathrm{~h}$ of culture in contact with the unmodified and vehicle-modified microneedles. The E. coli, P. aeruginosa, and $S$. aureus cultures did not exhibit growth inhibition following incubation with the voriconazole-modified microneedles. The $C$. albicans culture exposed to the voriconazole-modified microneedles demonstrated a zone of growth inhibition measuring $35 \mathrm{~mm}$ in diameter [Fig. 5(a)]. The visible zone of growth inhibition on the agar is indicative of the anti-C. albicans functionality of the voriconazole-modified microneedles.

It is interesting to note that the microneedle arrays that were modified by PMVE/MA copolymer-containing vehicle and voriconazole solutions did not show antibacterial activity. Previous work by Boehm et al. has indicated that microneedles made from Gantrez AN-169BF exhibit antibacterial properties. ${ }^{5}$ In addition, maleic acid and copolymers containing maleic anhydride have been shown to exhibit antimicrobial properties against Enterococcus faecalis, ${ }^{5,46}$ S. aureus, ${ }^{5,46,47}$ C. albicans, ${ }^{46,47}$ E. coli, ${ }^{5,47}$ and Bacillus subtilis. ${ }^{5}$ It should be noted that the previous study by Boehm et al. involved an entire microneedle device that was fabricated out of Gantrez AN-169BF and that the current study involves an Gantrez AN-169BF-containing microneedle coating; ${ }^{5}$ the microneedle in the previous study contained a much larger quantity of PMVE/MA than the microneedle in the current study. The inclusion of voriconazole in the inkjet printing-modified solution did impart antifungal activity to the microneedles against the $C$. albicans.

It should be noted that the number of printed layers can be increased in order to enlarge the voriconazole dosage without modifying the size of the microneedle array. The current microneedle array covers a surface area of roughly $12 \mathrm{~mm}^{2}$; several microneedle arrays could be used in a single step to treat a larger surface area. In addition, the dosage can be increased by treating a single site with several microneedle arrays.
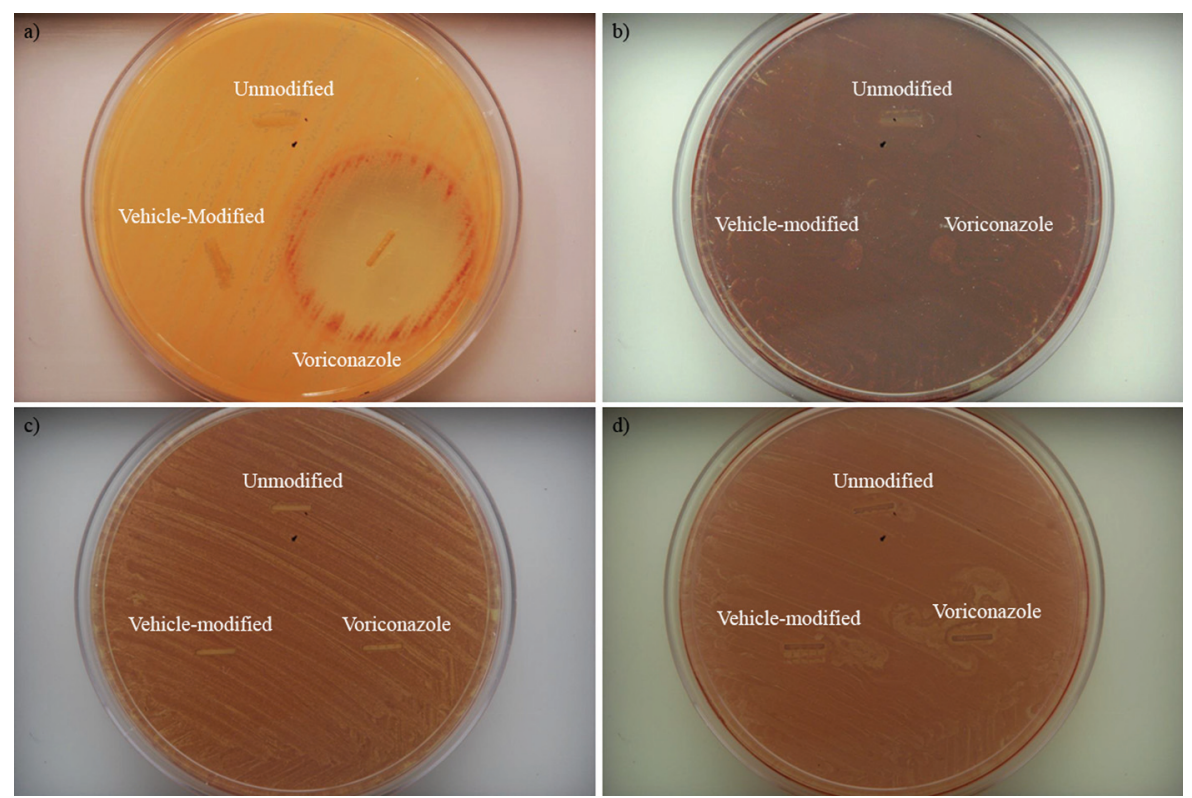

FIG. 5. Agar plates of microbial cultures following $24 \mathrm{~h}$ exposure to unmodified, vehicle-modified, and voriconazole-modified PGA microneedle arrays (a) $C$. albicans, (b) E. coli, (c) P. aeruginosa, and (d) S. aureus. A zone of growth inhibition is seen in (a) C. albicans at the insertion site of the voriconazolemodified microneedle array. 


\section{CONCLUSIONS}

In this study, microneedle arrays were fabricated using a combination of injection molding and drawing lithography; the sharpened microstructures created using this approach were capable of penetrating porcine skin samples. A piezoelectric inkjet printing process was utilized to deposit drug coatings onto the surfaces of PGA microneedles. Visual observations and EDS spectral analysis confirmed repeatable deposition of drug coatings on the microneedle surfaces. FTIR analysis indicated maintenance of the expected chemical groups of the substrate and the inkjet printing-modified materials. An agar plating study confirmed the antimicrobial functionality of the voriconazole-modified microneedle arrays. These findings suggest that PGA microneedles with inkjet deposited antifungal drug coatings may serve as transdermal drug delivery vehicles for antifungal applications.

\section{ACKNOWLEDGMENTS}

The authors would like to acknowledge B. Andersen and C. Mooney for their assistance with Fourier transform infrared spectroscopy and scanning electron microscopy, respectively. The authors would also like to acknowledge support from Office of Naval Research Grant N00014-12-1-0641. The authors declare no financial or commercial conflict of interest.

${ }^{1}$ Y.-C. Kim, J.-H. Park, and M. R. Prausnitz, Adv. Drug Delivery Rev. 64, 1547 (2012).

${ }^{2}$ M. R. Prausnitz, S. Mitragotri, and R. Langer, Nat. Rev. Drug Discovery 3, 115 (2004).

${ }^{3}$ F. Chabri, K. Bouris, T. Jones, D. Barrow, A. Hann, C. Allender, K. Brain, and J. Birchall, Br. J. Dermatol. 150, 869 (2004).

${ }^{4}$ S. D. Gittard, A. Ovsianikov, B. N. Chichkov, A. Doraiswamy, and R. J. Narayan, Expert Opin. Drug Delivery 7, 513 (2010).

${ }^{5}$ R. D. Boehm, P. R. Miller, R. Singh, A. Shah, S. Stafslien, J. Daniels, and R. J. Narayan, Biofabrication 4, 011002 (2012).

${ }^{6}$ Y. Nir, A. Paz, E. Sabo, and I. Potasman, Am. J. Trop. Med. Hyg. 68, 341 (2003).

${ }^{7}$ E. L. Giudice and J. D. Campbell, Adv. Drug Delivery Rev. 58, 68 (2006).

${ }^{8}$ S. Haeberle, D. Hradetzky, A. Schumacher, M. Vosseler, S. Messner, and R. Zengerle, IFMBE Proc. 25, 359 (2010).

${ }^{9}$ Y. Zhang, K. Brown, K. Siebenaler, A. Determan, D. Dohmeier, and K. Hansen, Pharm. Res. 29, 170 (2012).

${ }^{10}$ W. Martanto, S. P. Davis, N. R. Holiday, J. Wang, H. S. Gill, and M. R. Prausnitz, Pharm. Res. 21, 947 (2004).

${ }^{11}$ Y. C. Kim, F. S. Quan, D. G. Yoo, R. W. Compans, S. M. Kang, and M. R. Prausnitz, Vaccine 27, 6932 (2009).

${ }^{12}$ J.-H. Park, M. G. Allen, and M. R. Prausnitz, J. Controlled Release 104, $51(2005)$.

${ }^{13}$ C. G. Ambrose and T. O. Clanto, Ann. Biomed. Eng. 32, 171 (2004).

${ }^{14}$ J. Braunecker, M. Baba, G. E. Milroy, and R. E. Cameron, Int. J. Pharm. 282, 19 (2004).
${ }^{15}$ G. Shen, H. C. Tsung, C. F. Wu, X. Y. Liu, X. Y. Wang, W. Liu, L. Cui, and Y. L. Cao, Cell Res. 13, 335 (2003).

${ }^{16}$ J. M. Lippmann and A. P. Pisano, Proc. IEEE Micro Electro Mech. Syst. 19, 262 (2006)

${ }^{17}$ F. Sammoura, J. J. Kang, Y.-M. Heo, T. S. Jung, and L. Lin, Microsyst. Technol. 13, 517 (2007)

${ }^{18} \mathrm{~S}$. Aoyagi, H. Izumi, Y. Isono, M. Fukuda, and H. Ogawa, Sens. Actuators, A 139, 293 (2007).

${ }^{19}$ K. Lee and H. Jung, Biomaterials 33, 7309 (2012).

${ }^{20}$ K. Lee, C. Y. Lee, and H. Jung, Biomaterials 32, 3134 (2011).

${ }^{21}$ K. Lee, J. D. Kim, C. Y. Lee, S. Her, and H. Jung, Biomaterials 32, 7705 (2011).

${ }^{22}$ K. Lee, H. C. Lee, D.-S. Lee, and H. Jung, Adv. Mater. 22, 483 (2010).

${ }^{23}$ C. G. Li, C. Y. Lee, K. Lee, and H. Jung, Biomed. Microdevices 15, 17 (2013).

${ }^{24}$ R. D. Boehm, P. R. Miller, S. L. Hayes, N. A. Monteiro-Riviere, and R. J. Narayan, AIP Adv. 1, 022139 (2011).

${ }^{25}$ R. D. Boehm, P. R. Miller, W. A. Schell, J. R. Perfect, and R. J. Narayan, JOM 65, 525 (2013).

${ }^{26}$ R. D. Boehm, P. R. Miller, J. Daniels, S. Stafslien, and R. J. Narayan, Mater. Today 17, 247 (2014).

${ }^{27}$ W. C. Oliver and G. M. Pharr, J. Mater. Res. 7, 1564 (1992).

${ }^{28}$ NCCLS, Method for antifungal disk diffusion susceptibility testing of yeasts; approved guideline. NCCLS document M44-A. Wayne, PA, 2004.

${ }^{29}$ T. R. R. Singh, P. A. McCarron, A. D. Woolfson, and R. F. Donnelly, J. Appl. Polym. Sci. 112, 2792 (2009).

${ }^{30}$ The Infrared Spectroscopy Committee of the Chicago Society for Coatings Technology, An infrared spectroscopy atlas for the coatings industry. Federation of Societies for Coatings Technology, Philadelphia, PA, 1980, p. 269

${ }^{31}$ S. Mecham, A. Sentman, and M. Sambasivam, J. Appl. Polym. Sci. 116, 3265 (2010)

${ }^{32}$ Y. Li and P. I. Lee, Int. J. Pharm. 383, 45 (2010).

${ }^{33}$ A. Pandey, G. C. Pandey, and P. B. Aswath, J. Mech. Behav. Biomed. Mater. 1, 227 (2008).

${ }^{34}$ G. Kister, G. Cassanas, and M. Vert, Spectrochim. Acta, Part A 53, 1399 (1997).

${ }^{35}$ A. W. T. Shum and A. F. T. Mak, Polym. Degrad. Stab. 81, 141 (2003).

${ }^{36}$ M. Epple and O. Herzberg, J. Biomed. Mater. Res. Part A 43, 83 (1998).

${ }^{37}$ B. Sinha, B. Mukherjee, and G. Pattnaik, Nanomed. - Nanotechnol. Biol. Med. 9, 94 (2013).

${ }^{38}$ W. Xiang-Gen, Y. Li-Na, X. Meng, and J. Hao-Ran, J. Pharm. Sci. 100, 1745 (2011).

${ }^{39}$ N. Mozhzhukhina, L. P. Méndez De Leo, and E. J. Calvo, J. Phys. Chem. C 117, 18375 (2013)

${ }^{40}$ N. Scoutaris, M. R. Alexander, P. R. Gellert, and C. J. Roberts, J. Controlled Release 156, 179 (2011).

${ }^{41}$ N. Sandler, A. Määttänen, P. Ihalainen, L. Kronberg, A. Meierjohann, T. Viitala, and J. Peltonen, J. Pharm. Sci. 100, 3386 (2011).

${ }^{42}$ C. S. Kolli and A. K. Banga, Pharm. Res. 25, 104 (2008).

${ }^{43}$ M. I. Haq, E. Smith, D. N. John, M. Kalavala, C. Edwards, A. Anstey, A. Morrissey, and J. C. Birchall, Biomed. Microdevices 11, 35 (2009).

${ }^{44}$ G. Li, A. Badkar, H. Kalluri, and A. K. Banga, J. Pharm. Sci. 99, 1931 (2010).

${ }^{45}$ R. F. Donnelly et al., Pharm. Res. 28, 41 (2011)

${ }^{46}$ N. V. Ballal, P. P. Yegneswaran, K. Mala, and K. Seetharama, Oral Surg. Oral Med. Oral Pathol. Oral Radiol. Endod. 112, 696 (2011).

${ }^{47}$ H. A. Abd El-Rehim, A. E. Ali, T. B. Mostafa, and H. A. Farrag, Eur. Polym. J. 40, 2203 (2004) 